\title{
q-Completely monotonic and q-Bernstein functions
}

\author{
CH. G. KOKOLOGIANNAKI AND V. KRASNIQI
}

\begin{abstract}
We introduce the $q$-Bernstein functions, for $0<q<1$, and give sufficient and necessary conditions for a function to belong to the class of $q$-Bernstein functions. For some classes of functions we give results concerning $q$-completely monotonic and $q$-Bernstein functions. The obtained results are $q$-analogue of known results.
\end{abstract}

\section{Subject Classification: 05A30}

Additional Key Words and Phrases: completely monotonic functions, Bernstein functions, $q$-completely monotonic, $q$-Bernstein functions, positive definite functions, negative definite functions

\section{PRELIMINARIES}

$q$-calculus in the last twenty years served as a bridge between mathematics and physics. The field has expanded explosively due to the fact that applications of basic hypergeometric series to the diverse subjects of combinatorics, quantum theory, number theory, statistical mechanics are constantly being covered [4].

For the convenience of the reader we recall $[1,3,4,5]$ some notations and definitions concerning $q$-calculus.

The theory of $q$-analogues of classical formulas and functions is based on the observation that

$$
\lim _{q \rightarrow 1^{-}} \frac{1-q^{a}}{1-q}=a
$$

where $a \in C$ and $q$ can be any real or complex number and is called base. The number $\frac{1-q^{a}}{1-q}$ is called the "basic" number $[a]$ and it was first introduced by Heine (1847). So, we write

$$
[a]=\frac{1-q^{a}}{1-q}
$$


and of course

$$
\lim _{q \rightarrow 1^{-}}[a]=a .
$$

Throughout this paper we assume that $q$ satisfies the condition $0<q<1$.

The $q$-analogue of the Pochhammer symbol is:

$[a]_{k}=[a][a+1] \ldots[a+k-1]=\frac{\left(1-q^{a}\right) \ldots\left(1-q^{a+k-1}\right)}{(1-q)^{k}}, k \in N$, and $[a]_{0}=1$.

For $a=1$, the equation (1.4) becomes

$$
[1]_{k}=[1][2] \ldots[k]=[k] !
$$

Also the $q$-analogue of the Gauss binomial coefficients $\left(\begin{array}{l}n \\ k\end{array}\right)$ is defined

$$
\left[\begin{array}{l}
n \\
k
\end{array}\right]=\frac{[n] !}{[k] ![n-k] !}
$$

There are two analogues given in $[3,4]$ for the exponential function:

$$
e_{q}(z)=\sum_{n=0}^{\infty} \frac{z^{n}}{[n] !} \quad \text { and } \quad E_{q}(z)=\sum_{n=0}^{\infty} q^{\frac{n(n-1)}{2}} \frac{z^{n}}{[n] !}
$$

where the series converges for $|z|<\frac{1}{1-q}$ and $z \in C$ correspondingly.

We have to mention here that

$$
E_{q}(z)=e_{1 / q}(z)
$$

The $q$ derivative of an arbitrary function $f(z)$ is defined by

$$
D_{q}(f(z))=\frac{f(q z)-f(z)}{q z-z}, \quad z \neq 0 .
$$

It is clear that if the function $f(z)$ is differentiable then $\lim _{q \rightarrow 1^{-}}\left(D_{q}(f(z))=\frac{d f(z)}{d z}\right.$.

The properties of the $D_{q}$ operator can be found in $[1,3,4,5]$.

Very easily one can verify that

$$
D_{q}\left(e_{q}(a z)\right)=a e_{q}(a z) \quad \text { and } \quad D_{q}\left(E_{q}(a z)\right)=a E_{q}(q a z)
$$


for $|a z|<\frac{1}{1-q}$ and for $a, z \in C$ respectively. Also, by using induction we can prove that

$$
D_{q}^{n}\left(e_{q}(a z)\right)=a^{n} e_{q}(a z), \quad n \in N
$$

and

$$
D_{q}^{n}\left(E_{q}(a z)\right)=a^{n} q^{\frac{n(n-1)}{2}} E_{q}\left(q^{n} a z\right), \quad n \in N
$$

In [2] it has been introduced the definition of $q$-completely monotonic function.

DEFINITION 1.1. A positive function $f$ is said to be $q$-completely monotonic $(q-C M)$, if it an infinitely $q$-differentiable function such that $(-1)^{n} D_{q}^{n} f(z) \geq 0$ for $n=0,1, \ldots, z \in R^{+}$.

THEOREM 1.2. If $f(z), g(z)$ are $q-C M$ functions then $a f(z)+b g(z)$ and $f(z) g(z)$ are also, where $a, b$ are nonnegative constants.

THEOREM 1.3. A function $f:[0, \infty) \rightarrow R$ satisfying $\lim _{z \rightarrow \infty} f(z)=0$ is $q-C M$, if and only if it is the q-Laplace transform of a positive and bounded function $g$ defined on $R^{+}$such that $f(z)=\int_{0}^{\infty} E_{q}(-z t) g(t) d_{q} t$.

We have to mention that the definition for $q$-CM functions as well as the theorems 1.1 and 1.2 are the $q$-analogue of the definition of CM functions as well as the known theorems [6] for CM functions. So for $q \rightarrow 1^{-}$we obtain the known results.

REMARK 1.4. Using (1.10)-(1.12) if $a<0$ we can easily prove that the functions $e_{q}(a z)$ for $|z|<\frac{1}{1-q}$ and $E_{q}(a z)$ for $z \in C$ are $q-C M$.

In this paper we'll give some results concerning the $q$-CM, $q$-Bernstein functions and the positive and negative definite functions on the half-line.

The $q$-Bernstein functions are $q$-analogue of Bernstein functions, so we think that in this point it is necessary to recall [7] the definition of Bernstein functions and a basic theorem concerning them, as well as the definition of the positive and negative definite functions on the half-line. 
DEFINITION 1.5. A function $f:[0, \infty) \rightarrow R^{+}$is called Bernstein function if it has derivatives of all orders and $f^{\prime}$ is $C M$.

THEOREM 1.6. A function $f:[0, \infty) \rightarrow R^{+}$is a Bernstein function if and only if $f$ can be written in the form

$$
f(z)=a z+b+\int_{0}^{\infty}\left(1-e^{-t z}\right) \mu(d t)
$$

for $a, b \geq 0$ and $\mu$ is a Radon measure such that $\int_{0}^{\infty} \min (1, t) \mu d t<+\infty$.

DEFINITION 1.7. A function $f:[0,+\infty) \rightarrow C$ is positive definite if $\sum_{i, j=1}^{n} f\left(x_{i}+x_{j}\right) c_{i} \overline{c_{j}} \geq 0$ for $n \in N, \quad x_{i} \in[0,+\infty), i=1, \ldots, n$ and $c_{i} \in C$, $i=1, \ldots n$.

DEFINITION 1.8. A function $f:[0,+\infty) \rightarrow C$ is negative definite if it is hermitian $f(x)=\overline{f(x)}$ and $\sum_{i, j=1}^{n}\left(f\left(x_{i}\right)+\overline{f\left(x_{j}\right)}-f\left(x_{i}+x_{j}\right) c_{i} \overline{c_{j}} \geq 0\right.$ for $n \in$ $N, x_{i} \in[0,+\infty), i=1, \ldots, n$ and $c_{i} \in C, i=1, \ldots n$.

\section{MAIN RESULTS}

DEFINITION 2.1. A positive function $f$ on $[0,+\infty)$ is called $q$-Bernstein function if it is infinitely $q$-differentiable and $(-1)^{n-1} D_{q}^{n}(f(z)) \geq 0$ for $n=$ $0,1,2, \ldots$

It is obvious that a function $f$ non-negative and infinitely $q$-differentiable on $[0, \infty)$ is $q$-Bernstein function if and only if $D_{q}(f(z))$ is $q$-CM.

THEOREM 2.2. Let $f_{i}(z), i=1,2, \ldots, n$ be $q$-Bernstein function and $a_{i}>$ $0, i=1,2, \ldots, n$. Then $\sum_{i=1}^{n} a_{i} f_{i}(z)$ and $\prod_{i=1}^{n} f_{i}(z)$ are also q-Bernstein functions.

PROOF. It follows directly from the definition and the $q$-Leibnitz formula.

THEOREM 2.3. A function $f:[0, \infty) \rightarrow R^{+}$is a $q$-Bernstein function if and only if it admits the representation 


$$
f(z)=a z+b+\int_{0}^{\infty}\left(1-E_{q}(-t z)\right) \mu\left(d_{q}(t)\right)
$$

for $a, b \geq 0$ and $\mu d_{q}(t)$ is a positive measure on $[0, \infty)$.

PROOF. Assume that $f$ is a $q-$ Bernstein function. Then $D_{q}(f(z))$ is $q-$ CM. According to Theorem 1.2 there exists a measure $v$ on $[0, \infty)$ such that for all $z>0$

$$
D_{q}(f(z))=\int_{0}^{\infty} E_{q}(-z t) v\left(d_{q}(t)\right)
$$

Let $a=v(0)$. Then

$$
\begin{aligned}
f(z)-f(0+) & =\int_{0}^{z} D_{q}(f(y)) d_{q} y=a z+\int_{0}^{z} \int_{0}^{\infty} E_{q}(-y t) v\left(d_{q}(t)\right) d_{q}(y) \\
& =a z+\int_{0}^{\infty} \frac{1-E_{q}(-z t)}{t} v\left(d_{q}(t)\right) .
\end{aligned}
$$

Write $b=f(0+)$ and define $\mu\left(d_{q}(t)\right)=\frac{v\left(d_{q}(t)\right)}{t}$. Thus we completed the necessary condition. Conversely, since $f$ is $q$-CM it is $q$-differentiable and the $D_{q}(f(z))$ is given by

$$
D_{q}(f(z))=a+\int_{0}^{\infty} E_{q}(-z t) t \mu\left(d_{q}(t)\right)=\int_{0}^{\infty} E_{q}(-z t) v\left(d_{q}(t)\right)
$$

where $v\left(d_{q}(t)\right)=q t \mu\left(d_{q}(t)\right)+a \delta\left(d_{q}(t)\right)$,

$$
\int_{0}^{\infty} E_{q}(-z t) \delta\left(d_{q}(t)\right)=a
$$

and $\delta$ is the Dirac mass at point 0. From (2.5) we conclude that the function $D_{q}(f(z))$ is $q$-CM, so $f$ is a $q$-Bernstein function.

PROPOSITION 2.4. Let $f$ be a positive function on $(0, \infty)$ and $E_{q}(-k f(z))$, $k>0$, is q-CM function. Then $f$ is q-Bernstein function. 
PROOF. The series $E_{q}(-k f(z))=\sum_{j=0}^{\infty} \frac{q^{\frac{n(n-1)}{2}}(-1)^{j} k^{j}}{[j] !}(f(z))^{j}$ and all it's $q$-derivatives converge uniformly therefore we can calculate $D_{q}^{n} E_{q}(-k f(z))$ by termwise $q$-differentiation. Since $E_{q}(-k f(z))$ is $D_{q}$ completely monotonic we have:

$$
0 \leq(-1)^{n} D_{q}^{n} E_{q}(-k f(z))=\sum_{j=1}^{\infty} \frac{q^{n(n-1) 2} k^{j}}{[j] !}(-1)^{n+j} D_{q}^{n}\left((f(z))^{j}\right)
$$

Dividing by $k>0$ and letting $k \rightarrow 0$ we see $0 \leq(-1)^{n+1} D_{q}^{n}(f(z))$. The proof is completed.

PROPOSITION 2.5. If the function $f$ is $q$-CM then it is positive definite.

PROOF. Since $f$ is $q$-CM from theorem 1.3 it admits the representation

$$
f(z)=\int_{0}^{\infty} E_{q}(-z t) \mu\left(d_{q}(t)\right)
$$

and the sum in the definition 1.7 can be written:

$$
\sum_{i, j=1}^{n} f\left(z_{i}+z_{j}\right) c_{i} \overline{c_{j}}=\sum_{i, j=1}^{n} \int_{0}^{\infty} E_{q}\left(-t\left(z_{i}+z_{j}\right)\right) \mu\left(d_{q}(t)\right) c_{i} \overline{c_{j}}
$$

and using the fact that the equality

$$
E_{q}(a x+b y)=E_{q}(a x) E_{q}(b y)
$$

holds [1], the above sum becomes:

$$
\begin{aligned}
\sum_{i, j=1}^{n} f\left(z_{i}+z_{j}\right) c_{i} \overline{c_{j}}= & \int_{0}^{\infty}\left(\sum_{i=1}^{n} \sum_{j=1}^{n} E_{q}\left(-t\left(z_{i}\right) c_{i} \overline{E_{q}\left(-t\left(z_{j}\right) c_{j}\right.}\right) \mu\left(d_{q}(t)\right)=\right. \\
& \int_{0}^{\infty} \mid E_{q}\left(-\left.t\left(z_{i}\right) c_{i}\right|^{2} \mu\left(d_{q}(t)\right) \geq 0\right.
\end{aligned}
$$

so we get the desired result. 
REMARK 2.6. The above theorems and propositions are the q-analogue of theorems proved in [7].

PROPOSITION 2.7. Let $f$ be a positive increasing (or decreasing) function for $z \in[0, \infty)$. Then $f$ is $q$-increasing (or q-decreasing) function, which means that $D_{q}(f(z))>0\left(\right.$ or $\left.D_{q}(f(z))<0\right)$.

PROOF. The proof follows immediately, because for $0<q<1$ we have $z>q z$ and since $f^{\prime}(z)>0$ (or $\left.f^{\prime}(z)<0\right)$ from $(1.10)$ it follows that $D_{q}(f(z))>$ $0\left(\right.$ or $\left.D_{q}(f(z))<0\right)$.

THEOREM 2.8. Let $f:[0, \infty) \rightarrow R^{+} a C^{\infty}$ CM function. Then the function $f$ is $q-C M$.

PROOF. We have to prove that if $(-1)^{n} f^{(n)}(z) \geq 0$ then $(-1)^{n} D_{q}^{n}(f(z)) \geq$ $0, n \in N$. We'll prove it using the induction method.

For $n=1$ it holds, because: if $(-1) f^{\prime}(z)>0$ or $f^{\prime}(z)<0$ then from proposition 2.7 it follows that $D_{q}(f(z))<0$ or $(-1) D_{q}(f(z))>0$.

We assume that for $n=1,2, \ldots, k$ the desired result holds, that is if $(-1)^{k} f^{(k)}(z) \geq 0$ then $(-1)^{k} D_{q}^{k}(f(z)) \geq 0$. We will prove that it holds also for $n=k+1$, that is if $(-1)^{k+1} f^{(k+1)}(z) \geq 0$ then $(-1)^{k+1} D_{q}^{k+1}(f(z)) \geq 0$.

Indeed, if $f^{(k+1)}(z)>0$ then $f^{(k)}(z)$ increases, so $D_{q}^{k} f(z)>0$ and using proposition 2.7, $D_{q}^{k+1}(f(z))=D_{q}\left(D_{q}^{k} f(z)\right)>0$ and analogous if $f^{(k+1)}(z)<0$ then $f^{(k)}(z)$ decreases, so $D_{q}^{k} f(z)<0$ and using proposition $2.7, D_{q}^{k+1}(f(z))=$ $D_{q}\left(D_{q}^{k} f(z)\right)<0$. So, the desired result holds for every $n \in N$.

THEOREM 2.9. Let $f:[0, \infty) \rightarrow R^{+} a C^{\infty}$ Bernstein function. Then the function $f$ is q-Bernstein function.

PROOF. The proof is similar as in previous theorem, using the definition of Bernstein functions and proposition 2.7.

REMARK 2.10. It is obvious that the converse of the theorems 2.8 and 2.9 does not holds for every positive function $f$. 
For $C^{\infty}$ appropriate functions $f$ and $g$, the following propositions are the $q$-analoque of known [7], [8] and are derived very easily, according to the theorems 2.8 and 2.9 .

PROPOSITION 2.11. Let $f$ be an absolutely monotonic function (that is $f^{(n)}(z) \geq 0$ for $\left.z \in[0, \infty), n \in N_{0}\right)$ and $g$ be a $C^{\infty} C M$. Then the function $f \circ g$ is $q-C M$.

PROPOSITION 2.12. If $f$ is a positive $C^{\infty}$ function on $[0, \infty)$, then the following assertions are equivalent: (i) $f$ is $q$-Bernstein function, (ii) $g \circ f$ is $q$-CM for every function $g$ which is $q-C M$, (iii) $E_{q}(-k f)$ is $q$-CM for $k>0$.

PROPOSITION 2.13. Let $f$ be a $C^{\infty}$ q-Bernstein function. Then $f$ is negative definite function.

\section{REFERENCES}

[1] K.-S.Chung, W.-S.Chung, S.-T.Nam and H.-J.Kang, New $q$-derivative and $q$-Logarithm, International Journal of Theoretical Physics, 33, 10 (1994), 2019-2029.

[2] L.Dhaouadi, S.Guesmi, A. Fittouhi, $q$-Bessel positive definite and $D_{q}$-completely monotonic functions, Positivity, (2012) 16:107-129.

[3] T. Ernst, The history of q-calculus and a new method, U.U.D.M. Report 2000:16, ISSN 1101-3591, Department of Mathematics, Uppsala University, (2000).

[4] T. Ernst, A Comprehensive Treatment of q-Calculus, Birkhauser (2012)

[5] H. Exton, q-Hypergeometric functions and Applications, Ellis Horwood, Chichester, (1983).

[6] K.S.Miller, S.G.Samko, Completely monotonic functions, Integral Transforms and Special Functions, 12, 4 (2001) 389-402.

[7] R.L.Schilling, R.Song, Z.Vondracek, Bernstein functions,Theory and Application De Gruyter, (2010).

[8] H.M.Srivastava, S.Guo, F.Qi, Some properties of a class of functions related to completely monotonic functions, Computers and Mathematics with Applications 64 (2012) 1649-1654.

Chrysi G. Kokologiannaki

Department of Mathematics, University of Patras, 26500 Patras, Greece

e-mail: chrykok@math.upatras.gr

\section{Valmir Krasniqi}

Department of Mathematics and Computer Sciences,

University of Prishtina, Republic of Kosova

e-mail: vali.99@hotmail.com 